\title{
Farmacoterapia da obesidade: fármacos disponíveis no Brasil e perfis de eficácia e
}

\author{
segurança \\ Obesity pharmacotherapy: drugs available in Brazil and effectiveness and safety profiles \\ Farmacoterapia para obesidad: fármacos disponibles en Brasil y perfiles de eficacia y seguridad
}

Recebido: 28/07/2021 | Revisado: 02/08/2021 | Aceito: 17/09/2021 | Publicado: 18/09/2021

Kevellyn Bezerra Utta

ORCID: https://orcid.org/0000-0002-8849-701X Universidade Federal do Maranhão, Brasil

E-mail: kevellynutta@gmail.com

Débora Luana Ribeiro Pessoa

ORCID: https://orcid.org/0000-0002-9817-5647

Universidade Federal do Maranhão, Brasil

E-mail: debora.luana@ufma.br

\begin{abstract}
Resumo
A farmacoterapia da obesidade está em constante atualização e desenvolvimento de novos produtos e propostas, sendo por vezes visto como uma opção controversa, necessitando criteriosa avaliação para sua utilização, observando critérios como condição clínica do paciente e características do fármaco a ser utilizado, fazendo-se necessário o conhecimento sobre seus perfis de ação e segurança relacionados ao seu uso. O objetivo foi identificar e sistematizar os dados mais recentes presentes da literatura sobre os principais fármacos utilizados no tratamento da obesidade no Brasil e suas reações adversas. Foi realizada revisão integrativa de artigos publicados em português, inglês e espanhol, disponíveis na íntegra nas bases LILACS, BIREME (BVS) e MedLine. Utilizou-se os descritores "terapia medicamentosa" AND "obesidade" para a base LILACS, "terapia farmacológica" AND "obesidade" para a base Medline/Pubmed e "fármacos" AND "antiobesidade" para a base BIREME/BVS para artigos publicados no período de 2015 a 2020, sendo selecionados e analisados 15 estudos. Na base LILACs, MEDLINE/PUBMED e BIREME/BVS foram obtidos respectivamente 16, 49 e 305 artigos como resultados. Ao final de todas as análises e critérios de seleção, foram inclusos 15 artigos no presente estudo. A maioria dos medicamentos aprovados para o tratamento da obesidade no Brasil, demonstrou eficácia e segurança em ensaios clínicos. Foram observadas taxas semelhantes de eventos adversos entre orlistate, liraglutida e lorcaserina, com liraglutida possuindo maiores eventos gastrointestinais do grupo. Dentre as possibilidades de novos fármacos para a obesidade que apresentaram melhores resultados de perda de peso, foram exenatida e melatonina, com eficácia demonstrada em ensaios clínicos.
\end{abstract}

Palavras-chave: Tratamento farmacológico; Obesidade; Fármacos antiobesidade.

\begin{abstract}
The pharmacological treatment of obesity is constantly updated and the development of new products and proposals is often seen as a controversial option that requires a careful evaluation for its use, both with regard to the patient's clinical condition and the general characteristics of the drug. to be used, making it necessary to know about its action and safety profiles related to its use. The objective is identify and systematize the most recent data present in the literature on the main drugs used in the treatment of obesity in Brazil and their adverse reactions. Integrative review of articles published in Portuguese, English and Spanish, available in full on LILACS, BIREME (BVS) and MedLine databases. The keywords "drug therapy" AND "obesity" were used for the LILACS database, "pharmacological therapy" AND "obesity" for the Medline / Pubmed database and "drugs" AND "anti-obesity" for the BIREME / VHL database for articles published in the period from 2015 to 2020, 15 studies were selected and analyzed. Results: in LILACs, MEDLINE / PUBMED and BIREME / VHL databases, 16, 49 and 305 articles were obtained, respectively, as results. At the end of all analyzes and selection criteria, 15 articles were included in the present study. Most of the drugs approved for the pharmacotherapy of obesity in Brazil have demonstrated in clinical trials well-established efficacy in weight loss. Similar rates of adverse events were observed between orlistat, liraglutide and lorcaserin, with liraglutide having the largest gastrointestinal events in the group. In the study carried out, the possibilities of new pharmacological treatments for obesity that showed greater strength in the results of weight loss, were exenatide and melatonin, with efficacy demonstrated in clinical trials.
\end{abstract}

Keywords: Pharmacological treatment; Obesity; Anti-obesity drugs.

\section{Resumen}

La farmacoterapia de la obesidad se actualiza constantemente y el desarrollo de nuevos productos y propuestas, siendo vista en ocasiones como una opción controvertida, requiriendo una cuidadosa evaluación para su uso, mirando 
criterios como el estado clínico del paciente y las características del fármaco a usar, haciendo si es necesario, conocimiento de su actuación y perfiles de seguridad relacionados con su uso. El objetivo es identificar y sistematizar los datos más recientes presentes en la literatura sobre los principales fármacos utilizados en el tratamiento de la obesidad en Brasil y sus reacciones adversas. Revisión integradora de artículos publicados en portugués, inglés y español, disponibles íntegramente en las bases de datos LILACS, BIREME (BVS) y MedLine. Las palabras clave "farmacoterapia" Y "obesidad" se utilizaron para la base de datos LILACS, "terapia farmacológica" Y "obesidad" para la base de datos Medline / Pubmed y "fármacos" Y "anti-obesidad" para la base de datos BIREME / VHL para los artículos publicados. en el período de 2015 a 2020, se seleccionaron y analizaron 15 estudios. En las bases de datos LILACS, MEDLINE / PUBMED y BIREME / VHL se obtuvieron 16, 49 y 305 artículos, respectivamente, como resultados. Al final de todos los análisis y criterios de selección, se incluyeron 15 artículos en el presente estudio. La mayoría de los medicamentos aprobados para el tratamiento de la obesidad en Brasil han demostrado eficacia y seguridad en ensayos clínicos. Se observaron tasas similares de eventos adversos entre orlistat, liraglutida y lorcaserina, y la liraglutida tuvo los eventos gastrointestinales más importantes del grupo. Entre las posibilidades de nuevos fármacos para la obesidad que mostraron mejores resultados en la pérdida de peso, se encontraban la exenatida y la melatonina, con eficacia demostrada en ensayos clínicos.

Palabras clave: Tratamiento farmacológico; Obesidad; Medicamentos contra la obesidade.

\section{Introdução}

De acordo com a Organização Mundial de Saúde (OMS), a obesidade é definida como acúmulo excessivo e anormal de gordura no organismo, alcançando níveis capazes de comprometer a saúde em diversas faixas etárias, influenciando a qualidade de vida de crianças e adultos, e estando envolvida em consequências psicológicas e sociais (Costa \& Duarte, 2017).

O sobrepeso e a obesidade constituem o segundo fator de risco mais importante para a carga global de doenças, e estão associados com várias doenças crônicas não transmissíveis - DCNT, como doenças cardiovasculares, diabetes, câncer de cólon, de reto e de mama, cirrose, entre outras (ANS, 2017).

O avanço destas condições tem se dado de forma acelerada em todo o mundo nos últimos anos e no Brasil, a situação não é diferente. Em pesquisa realizada pelo IBGE, a proporção de obesos na população com 20 anos ou mais de idade mais que dobrou no país entre 2003 e 2019, passando de 12,2\% para 26,8\%. Nesse período, a obesidade feminina subiu de $14,5 \%$ para $30,2 \%$, enquanto a obesidade masculina passou de $9,6 \%$ para $22,8 \%$. Além disso, em 2019, uma em cada quatro pessoas de 18 anos ou mais anos de idade no Brasil estava obesa, o equivalente a 41 milhões de pessoas. Já o excesso de peso atingia 60,3\% da população de 18 anos ou mais de idade, o que corresponde a 96 milhões de pessoas, sendo 62,6\% das mulheres e $57,5 \%$ dos homens (IBGE, 2020).

Atualmente, o índice de massa corporal (IMC) é o indicador epidemiológico mais utilizado para o diagnóstico do sobrepeso e da obesidade. A equação proposta por Quetelet em 1835 considera o peso dividido pela altura ao quadrado. A faixa de peso de IMC considerada normal varia de 19 a 24,9 kg/m2. Pessoas com IMC de 25 a $30 \mathrm{~kg} / \mathrm{m} 2$ são consideradas acima do peso (sobrepeso), enquanto aquelas entre 30 a $34,9 \mathrm{~kg} / \mathrm{m} 2$ já são classificadas como obesidade I, 35 a $39,9 \mathrm{~kg} / \mathrm{m}^{2}$ classificam como obesidade II, e classificam como obesidade III os que se enquadram $\geq 40 \mathrm{~kg} / \mathrm{m}$ (Radaelli; Pedroso \& Medeiros, 2016).

O tratamento de pacientes obesos e com sobrepeso baseia-se em mudanças comportamentais, dieta e exercícios, com ou sem farmacoterapia ou cirurgia bariátrica, visando perder peso e diminuir fatores de risco (Lucchetta, et al. 2017).

Cruz e colaboradores (2013) afirmam que a obesidade é uma doença crônica e quando apenas mudanças no estilo de vida não produzem efeito esperado e/ou quando mais doenças estão relacionadas ou ainda quando houver predisposição a doenças, faz-se necessário o uso de medicamentos como tratamento adicional, pois a não utilização do fármaco pode agravar o quadro colocando em risco a vida do paciente. Também é importante frisar que o tratamento medicamentoso não cura a obesidade, mas pode controlar a doença e diminuir as comorbidades (Costa \& Duarte, 2017).

Nos casos em que a resposta ao tratamento é satisfatória, a manutenção da terapêutica medicamentosa por longo tempo deve ser considerada. No entanto, se as metas mencionadas não forem atingidas, ou se o paciente começar a recuperar o 
peso perdido, na vigência do tratamento farmacológico, orienta-se a substituição do medicamento. O aumento das doses acima das máximas recomendadas agrava os efeitos adversos dos fármacos, sem beneficiar o paciente. É importante frisar que não existe droga $100 \%$ eficaz, nem totalmente segura (SBEM, 2010).

De acordo com a ANVISA, no ano de 2011, os medicamentos sibutramina, anfepramona, femproporex e mazindol foram proibidos no Brasil em virtude dos resultados de um estudo realizado pela European Medicines Agency (EMA), órgão equivalente à ANVISA na União Europeia, que discutiu a eficácia e segurança desses fármacos, especialmente em relação à sibutramina, sendo esta reavaliada, ficando então demonstrado que seu benefício era maior que o seu risco, desde que utilizada adequadamente e para determinados perfis de pacientes. A partir de então, o controle sobre a sibutramina foi reforçado com a criação de uma receita especial para prescrição e comercialização do produto (ANVISA, 2018).

Em 2014 ocorreu a suspensão da proibição da produção, comercialização e consumo de anfepramona, femproporex e mazindol pela câmara dos deputados no Brasil, culminando na decisão por autorizá-los de forma definitiva no ano de 2017, desde que respeitado o critério de prescrição médica (Silva; Rodrigues \& Bonelli, 2019).

Atualmente, o registro dos medicamentos anfepramona, femproporex e mazindol, encontra-se cancelado, porém, é importante frisar que eles não estão proibidos, onde qualquer empresa da indústria farmacêutica pode pedir o registro das substâncias no Brasil, visto que não estão protegidas por patentes. Desta forma, estão atualmente autorizados e assegurados pela ANVISA no Brasil para tratar a obesidade, os fármacos: sibutramina, orlistate, cloridrato de lorcaserina e liraglutida (ANVISA, 2018).

A partir do presente estudo será possível identificar os principais fármacos disponíveis no Brasil para o tratamento da obesidade, bem como, seus mecanismos de ação e as principais reações adversas relacionadas ao seu uso objetivando integrar o amplo espectro de informação atualmente disponível na literatura acerca desse tema, bem como facilitar a identificação por parte dos profissionais de saúde, de possíveis alterações orgânicas decorrentes de sua utilização pela população.

\section{Metodologia}

Este estudo corresponde a uma revisão integrativa da literatura de caráter analítico a respeito da terapia farmacológica em vigência para o tratamento da obesidade. O presente trabalho segue os preceitos do estudo exploratório e qualitativo (Pereira et al.,2018).

A coleta de dados foi realizada no período de julho a outubro de 2020, sendo utilizadas para a pesquisa as bases de dados Literatura Latino-Americana e do Caribe em Ciências da Saúde (LILACS), Medical Literature Analysis and Retrieval System Online (MEDLINE) e Centro Latino-Americano e do Caribe de Informação em Ciências da Saúde (BIREME/BVS). Foi definido como critério de inclusão: artigos publicados entre os anos de 2015 e 2020, visando abranger o que há de mais atual disponível na literatura acerca do tema.

Nesta perspectiva, estiveram presentes as seguintes etapas:

\section{$1^{a}$ etapa-Fontes}

A seguir estão descritas as fontes utilizadas para a integração das informações de interesse para o presente trabalho:

a) Artigos científicos sobre a temática abordada, acessados nas bases de dados LILACS, MEDLINE e BIREME/BVS, publicados entre 2015 a 2020. foram utilizados artigos nacionais e internacionais disponíveis online em texto completo, em língua portuguesa, inglesa ou espanhola. Os seguintes descritores foram aplicados: "terapia medicamentosa" AND "obesidade" seguido de "fármacos" AND “antiobesidade". Em inglês: "drug therapy”, "obesity”, "drugs" e "anti-obesity".

Para a seleção das fontes, foram consideradas como critérios de inclusão trabalhos que abordem a terapia medicamentosa para o tratamento da obesidade e excluídas aqueles que não atendiam a temática de interesse para o estudo. 


\section{$2^{a}$ etapa - Coleta de Dados}

A coleta de dados passou pela seguinte premissa:

a) Leitura exploratória de todo o material selecionado (leitura rápida com o objetivo de identificar se o teor da obra é de interesse para o trabalho).

b) Leitura seletiva (leitura mais aprofundada das partes de interesse das obras selecionadas)

c) Registro das informações de interesse extraídas das fontes

\section{$3^{a}$ etapa - Análise e Interpretação dos Resultados}

Nessa etapa foi realizada leitura analítica do material selecionado, objetivando ordenar e sumariar as informações contidas nas fontes, possibilitando a obtenção de respostas ao questionamento levantado na pesquisa.

\section{$4^{a}$ etapa-Discussão dos Resultados}

Categorias emergentes da etapa anterior foram analisadas e discutidas a partir do referencial teórico relativo à temática do estudo.

\section{Resultados}

Na base LILACS foram utilizados os termos "tratamento farmacológico" e "obesidade", juntamente com o operador booleano "AND" para trabalhos publicados nos últimos 5 anos. Como resultados, foram obtidos 16 artigos na base, dos quais apenas 1 estava de acordo com o objetivo do estudo.

$\mathrm{Na}$ base MEDLINE/PUBMED, foram utilizados os termos: "farmacoterapia", "obesidade" e "tratamento da obesidade", juntamente com os operadores booleanos AND e OR, para trabalhos publicados nos últimos 5 anos. Como resultados, foram obtidos 49 artigos na base, os quais não foram selecionados artigos de acordo com o objetivo do estudo.

$\mathrm{Na}$ base BIREME/BVS, foram utilizados os termos "fármacos" AND "antiobesidade". Foram aplicados os seguintes filtros: tipos de estudo (ensaio clínico controlado, relatos de caso e estudos de prevalência) e idioma (português, inglês e espanhol). Foram encontrados 305 artigos, dos quais apenas 14 estavam de acordo com a temática do estudo. O Quadro 1 apresenta a síntese das informações explanadas acima.

Quadro 1: Resultados da busca em bases de dados.

\begin{tabular}{|l|l|l|l|l|}
\hline $\begin{array}{l}\text { SELEÇÃO DE } \\
\text { ESTUDOS }\end{array}$ & \multicolumn{3}{|c|}{$\begin{array}{l}\text { ARTIGOS POR BASE } \\
\text { DE DADOS }\end{array}$} & TOTAL \\
\hline & LILACS & MEDLINE/PubMed & BIREME/BVS & \\
\hline $\begin{array}{l}\text { Artigos encontrados na } \\
\text { busca inicial }\end{array}$ & 16 & 49 & 305 & 370 \\
\hline $\begin{array}{l}\text { Após leitura dos títulos } \\
\text { Após leitura dos } \\
\text { resumos }\end{array}$ & 1 & 0 & 62 & 63 \\
\hline $\begin{array}{l}\text { Após leitura do texto } \\
\text { completo }\end{array}$ & 1 & 0 & 21 & 22 \\
\hline
\end{tabular}

Fonte: Autores (2021). 
Após a seleção dos artigos conforme os critérios de inclusão previamente definidos, foram realizadas a seleção, leitura e interpretação dos textos, conforme exposto na Figura 1.

Figura 1: Fluxograma de seleção de seleção dos artigos para inclusão no estudo.

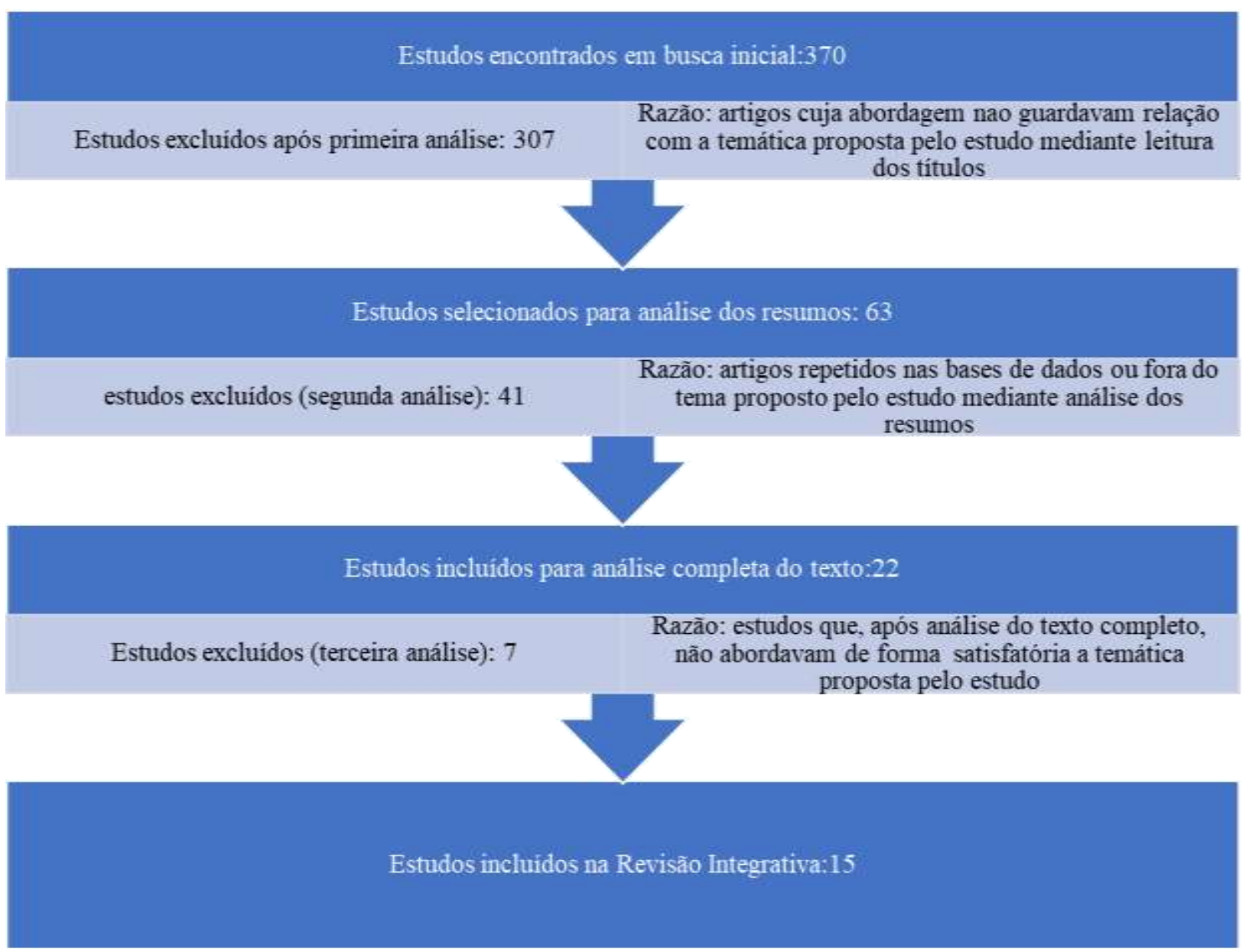

Fonte: Autores (2021).

Na sequência agruparam-se os temas mais abordados nas seguintes categorias: critérios para tratamento farmacológico da obesidade; terapia farmacológica atual, reações adversas relacionadas às classes farmacológicas e novos fármacos para o tratamento da obesidade.

Segue no Quadro 2 a categorização das informações obtidas dos artigos analisados.

Quadro 2: Categorização das informações obtidas dos artigos selecionados.

\begin{tabular}{|c|c|c|c|c|c|}
\hline $\begin{array}{l}\mathrm{N}^{\circ} \text { do } \\
\text { artigo }\end{array}$ & $\begin{array}{l}\text { Ano de } \\
\text { publicação }\end{array}$ & Título & $\begin{array}{l}\text { Tipo de estudo } \\
\text { (metodologia) }\end{array}$ & Resumo do artigo & Autores \\
\hline 01 & 2016 & $\begin{array}{l}\text { Massa gorda reduzida e } \\
\text { massa magra aumentada } \\
\text { em resposta a } 1 \text { ano de } \\
\text { tratamento } \quad \text { com } \\
\text { melatonina em mulheres } \\
\text { na pós-menopausa: um } \\
\text { ensaio randomizado } \\
\text { controlado por placebo }\end{array}$ & Ensaio clínico & $\begin{array}{l}\text { Este estudo buscou } \\
\text { determinar os efeitos da } \\
\text { melatonina na } \\
\text { composição corporal e no } \\
\text { metabolismo de lipídeos } \\
\text { e glicose em humanos. }\end{array}$ & $\begin{array}{lrr}\text { Anne Kristine } & \text { Amstrup , } \\
\text { Tanja Sikjaer } & \text { Steen B. } \\
\text { Pedersen } & , & \text { Lene } \\
\text { Heickendorff } & \text {, } & \text { Leif } \\
\text { Mosekilde } & \text { e } & \text { Lars } \\
\text { Rejnmark } & & \end{array}$ \\
\hline 02 & 2016 & $\begin{array}{l}\text { A eficácia e segurança do } \\
\text { Liraglutida } 3,0 \mathrm{mg} \text { para } \\
\text { controle de peso são }\end{array}$ & Análise post hoc & $\begin{array}{l}\text { Fez a análise de } \text { dados } \\
\text { agrupados de } \begin{array}{c}\text { cinco } \\
\text { ensaios }\end{array} \text { clínicos }\end{array}$ & $\begin{array}{l}\text { Ard, J; Cannon, A; Lewis, } \\
\text { C E; Lofton, H; Vang } \\
\text { Skjøth, T; Stevenin, B; Pi- }\end{array}$ \\
\hline
\end{tabular}




\begin{tabular}{|c|c|c|c|c|c|}
\hline & & $\begin{array}{l}\text { semelhantes } \\
\text { entre as raças: análise de } \\
\text { subgrupo na ESCALA e } \\
\text { ensaios randomizados de } \\
\text { fase II }\end{array}$ & & $\begin{array}{l}\text { randomizados, duplo- } \\
\text { cegos e controlados por } \\
\text { placebo compara a } \\
\text { eficácia e segurança de } \\
\text { Liraglutida } 3,0 \text { mg versus } \\
\text { placebo entre diferentes } \\
\text { subgrupos raciais }\end{array}$ & Sunyer, \\
\hline 03 & 2016 & $\begin{array}{l}\text { Eficácia de uma } \\
\text { combinação Orlistate- } \\
\text { Resveratrol para perda de } \\
\text { peso em indivíduos com } \\
\text { obesidade: um ensaio } \\
\text { clínico randomizado }\end{array}$ & Ensaio clínico & $\begin{array}{l}\text { Avaliou a eficácia de } \\
\text { uma combinação de } \\
\text { Orlistate-Resveratrol } \\
\text { (OR) em indivíduos com } \\
\text { obesidade ao longo de } \\
\text { um período de } 6 \text { meses. }\end{array}$ & $\begin{array}{l}\text { Marı Angelica Arzola- } \\
\text { Paniagua, Enrique Raul } \\
\text { Garcia-Salgado Lopez , } \\
\text { Cesar G. Calvo-Vargas e } \\
\text { Martha Guevara-Cruz }\end{array}$ \\
\hline 04 & 2016 & $\begin{array}{l}\text { Uma comparação de } \\
\text { novos } \\
\text { farmacológicos para ontes } \\
\text { tratamento da obesidade }\end{array}$ & $\begin{array}{ll}\text { Ensaio } & \text { clínico } \\
\text { controlado } & \end{array}$ & $\begin{array}{l}\text { Rever e comparar as } \\
\text { evidências dos ensaios } \\
\text { clínicos de fase } 3 \text { sobre } \\
\text { os } 4 \text { novos agentes } \\
\text { farmacológicos } \\
\text { lorcaserina, } \\
\text { naltrexona/bupropiona, } \\
\text { fentermina/topiramato e } \\
\text { liraglutida, aprovados } \\
\text { para o tratamento do } \\
\text { sobrepeso e da } \\
\text { obesidade. }\end{array}$ & $\begin{array}{l}\text { Nuffer, Wesley; Trujillo, } \\
\text { Jennifer M; Megyeri, Jade. }\end{array}$ \\
\hline 05 & 2016 & $\begin{array}{l}\text { Papel dos agonistas do } \\
\text { receptor do peptídeo } 1 \\
\text { semelhante ao glucagon } \\
\text { no tratamento da } \\
\text { obesidade }\end{array}$ & $\begin{array}{l}\text { Guia de prática } \\
\text { clínica / Estudo de } \\
\text { prevalência }\end{array}$ & $\begin{array}{l}\text { Os dados publicados } \\
\text { sobre os efeitos da perda } \\
\text { de peso dos agonistas do } \\
\text { receptor do peptídeo } \\
\text { semelhante ao glucagon } \\
1 \text { (GLP-1) foram } \\
\text { revisados, com foco em } \\
\text { dados de ensaios clínicos }\end{array}$ & $\begin{array}{l}\text { Isaacs, Diana; Prasad- } \\
\text { Reddy, Lalita; Srivastava, } \\
\text { Sneha Baxi }\end{array}$ \\
\hline 06 & 2017 & $\begin{array}{l}\text { Avaliação } \\
\text { farmacocinética de } \\
\text { drogas de Lorcaserina de } \\
\text { liberação prolongada } \\
\text { para o tratamento da } \\
\text { obesidade }\end{array}$ & $\begin{array}{ll}\text { Ensaio } & \text { clínico } \\
\text { controlado } & \end{array}$ & $\begin{array}{lr}\text { Avaliou } & \text { a } \\
\text { farmacocinética de } & \text { drogas de Lorcaserina de } \\
\text { liberação prolongada } \\
\text { para o tratamento da } \\
\text { obesidade }\end{array}$ & $\begin{array}{l}\text { Kathryn Hurren e Marissa } \\
\text { W. Dunham }\end{array}$ \\
\hline 07 & 2017 & $\begin{array}{l}\text { Coadministração de } \\
\text { Lorcaserina e Fentermina } \\
\text { para controle de peso: um } \\
\text { estudo piloto de } \\
\text { segurança randomizado } \\
\text { de } 12 \text { semanas }\end{array}$ & $\begin{array}{l}\text { Ensaio clínico } \\
\text { randomizado }\end{array}$ & 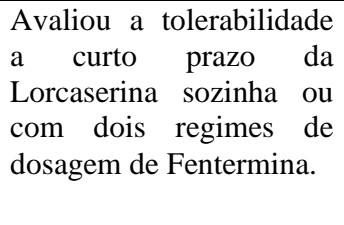 & $\begin{array}{l}\text { Steven R. Smith, W. } \\
\text { Timothy Garvey, Frank L. } \\
\text { Greenway, Sharon Zhou, } \\
\text { Randi Fain, Robert Pilson, } \\
\text { Ken Fujioka, } \\
\text { e Louis J. Aronne }\end{array}$ \\
\hline 08 & 2017 & 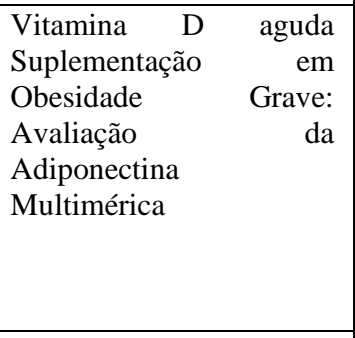 & $\begin{array}{l}\text { Ensaio clínico } \\
\text { controlado }\end{array}$ & $\begin{array}{l}\text { Avaliou os efeitos } \\
\text { agudos de } 600.000 \text { UI de } \\
\text { colecalciferol } \\
\text { administrados em } 12 \\
\text { individuos, sobre os } \\
\text { níveis de adiponectina de } \\
\text { baixo, médio e alto peso } \\
\text { molecular. }\end{array}$ & $\begin{array}{l}\text { Stefania Mai, Gillian E. } \\
\text { Walker, Roberta Vietti, } \\
\text { Stefania Cattaldo, Chiara } \\
\text { Mele, } \\
\text { Lorenzo Priano, Alessandro } \\
\text { Mauro, Gianni Bona, } \\
\text { Gianluca Aimaretti, } \\
\text { Massimo Chess e Paolo } \\
\text { Marzullo }\end{array}$ \\
\hline 09 & 2017 & $\begin{array}{l}\text { Segurança } \\
\text { neuropsiquiátrica com } \\
\text { Liraglutida } 3,0 \mathrm{mg} \text { para } \\
\text { controle de peso: } \\
\text { resultados de ensaios de } \\
\text { fase } 2 \text { e } 3 \text { a controlados } \\
\text { randomizados }\end{array}$ & $\begin{array}{l}\text { Ensaio clínico } \\
\text { randomizado }\end{array}$ & $\begin{array}{l}\text { Foram avaliados dados } \\
\text { de segurança } \\
\text { neuropsiquiátrica } \\
\text { combinados de todos os } \\
\text { ensaios clínicos } \\
\text { randomizados de fase } 2 \text { e } \\
\text { 3a duplo-cegos com } \\
\text { liraglutido } 3,0 \mathrm{mg} \text { post } \\
\text { hoc. }\end{array}$ & $\begin{array}{l}\text { PM O'Neil, VR Aroda, A. } \\
\text { Astrup, R. Kushner, DCW } \\
\text { Lau, TA Wadden, J. Brett } \\
\text {, A. Cancino, JPH Wilding. }\end{array}$ \\
\hline 10 & 2017 & $\begin{array}{l}\text { Efeitos da suplementação } \\
\text { de ácido }\end{array}$ & $\begin{array}{l}\text { Ensaio clínico } \\
\text { controlado }\end{array}$ & $\begin{array}{l}\text { Determinou os efeitos da } \\
\text { suplementação de ácido } \\
\text { graxo poliinsaturado n-3 } \\
\text { sobre os níveis de }\end{array}$ & $\begin{array}{l}\text { L. Payahoo , A. } \\
\text { Ostadrahimi , N. Farrin, e } \\
\text { Y. Khaje-Bishak }\end{array}$ \\
\hline
\end{tabular}




\begin{tabular}{|c|c|c|c|c|c|}
\hline & & $\begin{array}{l}\text { sérica, as sensações de } \\
\text { apetite e a ingestão de } \\
\text { energia e macronutrientes } \\
\text { em pessoas obesas: um } \\
\text { ensaio clínico } \\
\text { randomizado }\end{array}$ & & $\begin{array}{l}\text { Leptina sérica, sensações } \\
\text { de apetite e ingestão } \\
\text { alimentar em pessoas } \\
\text { obesas }\end{array}$ & \\
\hline 11 & 2018 & $\begin{array}{l}\text { Mudanças nos hormônios } \\
\text { da saciedade em resposta } \\
\text { ao tratamento com } \\
\text { Leptina em um paciente } \\
\text { com deficiência de } \\
\text { Leptina }\end{array}$ & Relato de caso & $\begin{array}{l}\text { Testou se o tratamento } \\
\text { com leptina afeta a } \\
\text { seculina, o peptídeo-1 } \\
\text { semelhante ao glucagon } \\
\text { (GLP-1), a grelina e a } \\
\text { secreção peptídica de } \\
\text { peptídeos intestinais } \\
\text { relacionados à saciedade } \\
\text { e a secreção de YY } \\
\text { (PYY) derivada do } \\
\text { cérebro. }\end{array}$ & $\begin{array}{l}\text { Roth, Christian L; von } \\
\text { Schnurbein, Julia; Elfers, } \\
\text { Clinton; Moss, Anja; } \\
\text { Wabitsch, Martin. }\end{array}$ \\
\hline 12 & 2018 & $\begin{array}{l}\text { Eficácia comparativa } \\
\text { observacional } \\
\text { tratamentos } \\
\text { farmacêuticos de } \\
\text { obesidade em } \\
\text { Health Administration }\end{array}$ & $\begin{array}{l}\text { Ensaio clínico } \\
\text { controlado / Estudo } \\
\text { de incidência }\end{array}$ & $\begin{array}{l}\text { Comparou a eficácia dos } \\
\text { medicamentos de } \\
\text { controle de peso usados } \\
\text { para auxiliar na perda de } \\
\text { peso na prática clínica do } \\
\text { mundo real na Veterans } \\
\text { Health Administration } \\
\text { (VHA). }\end{array}$ & Ted R. Grabarczyk \\
\hline 13 & 2018 & $\begin{array}{l}\text { Liraglutida e desfechos } \\
\text { cardiovasculares em } \\
\text { adultos com sobrepeso } \\
\text { ou obesidade: A post hoc } \\
\text { - análise de ensaios } \\
\text { clínicos randomizados } \\
\text { SCALE }\end{array}$ & Análise post hoc & $\begin{array}{l}\text { Avaliou se o risco } \\
\text { cardiovascular aumentou } \\
\text { com o tratamento com } \\
\text { Liraglutida. O resultado } \\
\text { composto primário desta } \\
\text { análise do tempo até o } \\
\text { evento foi a primeira } \\
\text { ocorrência de morte } \\
\text { cardiovascular, infarto do } \\
\text { miocárdio não fatal ou } \\
\text { acidente vascular } \\
\text { cerebral não fatal. }\end{array}$ & $\begin{array}{l}\text { MJ Davies, LJ Aronne, ID } \\
\text { Caterson, AB Thomsen , } \\
\text { PB Jacobsen e SP Marso }\end{array}$ \\
\hline 14 & 2019 & $\begin{array}{l}\text { Um ensaio randomizado, } \\
\text { duplo-cego, controlado } \\
\text { por placebo de } 6 \text { meses } \\
\text { de Exenatida semanal em } \\
\text { adolescentes } \\
\text { obesidade }\end{array}$ & $\begin{array}{l}\text { Ensaio clínico } \\
\text { randomizado }\end{array}$ & $\begin{array}{l}\text { Investigou o efeito da } \\
\text { liberação prolongada de } \\
\text { Exenatida no índice de } \\
\text { massa corporal (IMC) - } \\
\text { SDS como resultado } \\
\text { primário e no } \\
\text { metabolismo da glicose e } \\
\text { no risco } \\
\text { cardiometabólico }\end{array}$ & $\begin{array}{l}\text { D. Weghuber A. Forslund, } \\
\text { H. Ahlström }\end{array}$ \\
\hline 15 & 2020 & $\begin{array}{l}\text { Liraglutida } 3,0 \text { mg e } \\
\text { terapia comportamental } \\
\text { intensiva (IBT) para } \\
\text { obesidade na atenção } \\
\text { primária: o ensaio clínico } \\
\text { controlado SCALE IBT }\end{array}$ & Ensaio clínico & $\begin{array}{l}\text { O presente estudo } \\
\text { forneceu a primeira } \\
\text { avaliação randomizada e } \\
\text { controlada por placebo } \\
\text { de IBT, em combinação } \\
\text { com liraglutida } 3,0 \mathrm{mg} \text {, } \\
\text { conforme testado em um } \\
\text { estudo multisite com } \\
\text { pacientes principalmente } \\
\text { de clínicas de atenção } \\
\text { primária }\end{array}$ & $\begin{array}{l}\text { Jena Shaw Tronieri, Danny } \\
\text { Sugimoto, Michael Taulo } \\
\text { Lund, Pernille Auerbach, } \\
\text { Thomas A. Wadden } \\
\text { Camilla Jensen e Domenica } \\
\text { Rubino }\end{array}$ \\
\hline
\end{tabular}

Fonte: Autores (2021).

\section{Discussão}

Dos 15 artigos selecionados neste estudo que abordam aspectos relativos à farmacoterapia da obesidade, 7 destes referem-se a fármacos liberados atualmente pela ANVISA para tratamento da obesidade no Brasil, os quais serão discutidos neste trabalho, além de novas perspectivas dentro da terapêutica farmacológica antiobesidade. 


\subsection{Sibutramina}

A sibutramina enquadra-se no grupo dos fármacos simpatomiméticos. Aprovada no ano de 1997 para uso de longo prazo no tratamento da obesidade, sendo suspensa na Europa e EUA no ano de 2010 devido a verificação de riscos referentes a efeitos adversos da medicação (Silva; Rodrigues \& Bonelli, 2019).

\subsubsection{Mecanismo de ação}

Sua ação farmacológica se dá por meio do aumento da atividade noradrenérgica no hipotálamo e na periferia, mais especificamente através da inibição da recaptação de noradrenalina, serotonina e dopamina, responsáveis pelos efeitos anorexigênicos do fármaco, o aumento do gasto de energia e saciedade precoce (Silva; Rodrigues \& Bonelli, 2019).

No Brasil encontra-se disponível para comercialização, sendo classificado hoje como um medicamento sujeito a controle especial.

\subsubsection{Eficácia e segurança}

A sibutramina foi inicialmente estudada como uma possibilidade de tratamento antidepressivo, sendo testada em estudos de fase II, onde mostrou-se pouco eficaz. Em contrapartida, demonstrou impactos significativos na perda de peso, dando-se prosseguimento a ensaios clínicos para comprovar sua ação com essa finalidade. Verificou-se então que a sua utilização diminuiu a ingestão de alimentos e aumentou o gasto de energia, resultando em perda de peso sustentável e dependente da dose significativa, com 77\% dos participantes alcançando uma perda de peso de $\geq 5 \%$ em 6 meses, concomitante a melhorias nos índices de controle de lipídios e glicemia (Shettar; Patel \& Kidambi, 2017).

Seus principais efeitos adversos estão relacionados ao sistema cardiovascular. Grandes estudos clínicos separados encontraram pequenos aumentos médios na pressão sanguínea e no pulso, levando a um grande estudo de longo prazo, o estudo SCOUT - Sibutramine Cardiovascular Outcomes Trial (Gadde; Alpolzan \& Berthoud, 2018).

No estudo SCOUT, um ensaio clínico randomizado realizado com 11.000 pacientes obesos com alto risco de doenças cardiovasculares, analisando sibutramina versus placebo, foram verificados eventos cardiovasculares maiores (infarto agudo do miocárdio não fatal, acidente vascular cerebral, parada cardíaca ou morte cardiovascular) em que foi demonstrado um aumento do risco de ataques cardíacos e infartos do miocárdio neste grupo específico. O estudo foi interrompido após 3,4 anos e teve como resultado um desfecho primário com taxa de $11,2 \%$ no grupo da sibutramina e $10 \%$ no grupo do placebo, revelando um risco aumentado de eventos cardiovasculares adversos maiores com o tratamento com sibutramina em relação ao placebo em pacientes de alto risco, culminando na sua retirada do mercado em países como Estados Unidos e Canadá (Shettar; Patel \& Kidambi, 2017).

\subsection{Orlistate}

\subsubsection{Mecanismo de ação}

$\mathrm{O}$ orlistate trata-se de um inibidor da lipase pancreática que age reduzindo a absorção intestinal de gordura em cerca de um terço, estando disponível no mercado desde 1999. Esta classe de medicamentos altera a digestão da gordura ao inibir irreversivelmente as lipases pancreáticas e gástricas. Visto que a lipase pancreática é a principal enzima que decompõe os triglicerídeos da dieta, ao diminuir as lipases ativas no trato gastrointestinal, as gorduras da dieta não podem mais ser hidrolisadas em ácidos graxos livres absorvíveis e monogliceróis e são excretadas como triglicerídeos nas fezes (Shettar; Patel \& Kidambi, 2017).

De acordo com a análise dos estudos selecionados, identificou-se que, dentre os impactos que este composto produz no organismo, podemos citar uma mudança significativa na perda de peso corporal concomitante a melhora nos níveis da 
glicemia de jejum e na hemoglobina glicosilada.

\subsubsection{Eficácia e segurança}

No ensaio XENDOS (Xenical in prevention of diabetes in obese subjects study), 3.305 participantes obesos (21\% destes com pré-diabetes) foram randomizados para mudanças no estilo de vida e orlistate (120 mg) ou placebo 3 vezes ao dia e acompanhados por 4 anos. Durante o primeiro ano, a perda de peso foi maior no grupo tratado com orlistate em relação ao grupo tratado com placebo ( $10 \%$ x 6\%), porém Ohavendo leve recuperação de peso durante os anos subsequentes. Os participantes que receberam orlistate perderam ainda mais peso ao final de 4 anos versus placebo (6 $\mathrm{kg}$ [7\%] versus $3 \mathrm{~kg}$ [4\% abaixo do peso basal]). A perda de peso líquido com orlistate é de cerca de $3 \mathrm{~kg}$ em comparação com placebo no contexto de modificação do estilo de vida (Shettar; Patel \& Kidambi, 2017).

Seus principais efeitos adversos mencionados estão relacionados ao trato gastrointestinal e incluem principalmente esteatorréia, aumento da frequência de defecação, fezes líquidas, urgência fecal e flatulência. Foi identificado ainda um impacto sobre a absorção de vitaminas lipossolúveis e de ciclosporina (Arzola-Paniagua, 2016).

$\mathrm{O}$ orlistate é um dos primeiros medicamentos comercializados para perda de peso e, portanto, tem os maiores dados de segurança cardiovascular e pode ser usado para o tratamento de longo prazo da obesidade. Por causa dos efeitos colaterais gastrointestinais não é um medicamento popular. É o único medicamento aprovado pelo FDA para perda de peso em adolescentes menores de 12 anos (Gadde; Apolzan \& Berthoud, 2018).

\subsection{Liraglutida}

Durante a pesquisa foi possível verificar que, o maior contingente de artigos científicos que se enquadravam nesse estudo foi referente à liraglutida. Cinco deles traziam uma análise aprofundada sobre este fármaco, sendo observadas características como eficácia, segurança, diferentes impactos de ação entre as diferentes raças, desfechos cardiovasculares entre outros.

\subsubsection{Mecanismo de ação}

O GLP-1 (peptídeo semelhante ao glucagon 1) é um hormônio endógeno produzido principalmente pelas células enteroendócrinas L no íleo e cólon em resposta à ingestão alimentar. Os níveis plasmáticos de GLP-1 aumentam minutos após a refeição, estimulando a secreção de insulina, diminuindo a secreção de glucagon do pâncreas, diminuindo a gliconeogênese hepática e melhorando a sensibilidade à insulina. Os tecidos do sistema cardiovascular, sistema nervoso central e sistema gastrointestinal também contêm receptores de GLP-1, e este é conhecido por retardar o esvaziamento gástrico e promover saciedade. O GLP-1 atravessa a barreira hematoencefálica e, dentro do hipotálamo, afeta a regulação do apetite ao influenciar os sinais de saciedade. Também há evidências dos efeitos do GLP-1 sobre o gasto de energia que favorecem a oxidação de gordura em comparação com carboidratos. (Nuffer; Trujillo \& Megyeri, 2016).

\subsubsection{Eficácia e segurança}

No que diz respeito à eficácia, todos os estudos avaliados (Ard et al., 2016; Isaacs et al., 2016; O'neil et al., 2017; Grabarczyk, 2018; Davies et al., 2018; Wadden et al., 2020) evidenciaram a efetividade do tratamento com liraglutida 3 mg no manejo da obesidade, fato este observado a partir dos seguintes dados:

Em estudo multisite e controlado por placebo foi analisada a combinação terapia comportamental intensiva (TCI) com liraglutida 3,0 mg e TCI associada a placebo durante 56 semanas, sendo realizada uma avaliação randomizada com pacientes principalmente de clínicas de atenção primária. Foi observado neste estudo que em indivíduos com obesidade, o liraglutida- 
TCI foi superior ao placebo-TCI, reduzindo o peso corporal basal e produzindo um valor clinicamente significativo de $\geq 5 \%$ de perda de peso na semana 56, num percentual de 61,5\% para com liraglutida-TCI e 38,8\% para placebo-TCI (Wadden et al., 2020).

Com relação as reações adversas, este mesmo estudo evidenciou que as mais frequentes foram de natureza gastrointestinal, a maioria correspondendo a náuseas, sendo estas mais frequentes com a utilização de liraglutida associada a TCI quando em comparação com placebo (47,9\% versus $17,9 \%)$. A maioria dos eventos foi de gravidade leve ou moderada e ocorreu principalmente nas primeiras 4 a 8 semanas de tratamento. A frequência cardíaca aumentou em ambos os grupos, com um aumento numericamente maior com liraglutida-TCI em comparação com placebo-TCI (Wadden et al., 2020).

Em estudo de Ard et al. (2016) realizado para verificar a eficácia e segurança de liraglutida $3 \mathrm{mg}$ entre diferentes raças, foi visto que os efeitos da liraglutida no peso foi significativamente maior quando em comparação com o grupo placebo, sendo evidenciado pelo fato de que mais indivíduos perderam $\geq 5 \%,>10 \%$ e> $15 \%$ de seu peso corporal basal no grupo de liraglutida 3,0 mg em comparação ao grupo placebo nos subgrupos raciais. Os efeitos médios e categóricos da perda de peso não mostraram qualquer dependência do subgrupo racial, neste estudo.

Em cada subgrupo racial, os eventos relatados com mais frequência foram distúrbios gastrointestinais, que foram relatados mais frequentemente com liraglutida $3,0 \mathrm{mg}$ do que com placebo. A proporção de indivíduos que relataram eventos adversos gerais, eventos adversos graves e eventos que levaram à descontinuação foi semelhante para brancos, negros / afroamericanos, asiáticos e outros grupos raciais estudados (Ard et al., 2016).

Em revisão clínica realizada por Isaacs; Prasad-Reddy E Srivastava (2016) para verificar os efeitos dos análogos do GLP-1 sobre a perda de peso a partir de análise de dados de ensaios clínicos evidenciou que na maioria dos estudos realizados com pacientes obesos sem diabetes, os indivíduos obtiveram perda significativa de peso, bem como manutenção de perda de peso em $\geq 5 \%$ mediante o uso de liraglutida $3 \mathrm{mg}$ quando em comparação com placebo.

Os efeitos adversos descritos neste estudo, como os mais comuns dos agonistas do receptor de GLP-1 são gastrointestinais e incluem diarreia, náuseas e vômitos. Sugere-se que esses efeitos são mais prováveis de ocorrer com doses mais altas de agonistas do receptor de GLP-1 ou com aumentos abruptos da dose (Isaacs; Prasad-Reddy \& Srivastava, 2016).

Ao considerar efeitos adversos de liraglutida um estudo realizado para avaliação da segurança neuropsiquiátrica com uso de liraglutida em 5523 individuos obesos expostos à dosagem de $3 \mathrm{mg}$ e placebo, ambos associados a atividade física e dieta, foi observado que as taxas de depressão por meio de relatórios de eventos adversos foram igualmente baixas no grupo de liraglutida e o grupo do placebo. Nove $(0,3 \%)$ indivíduos com liraglutida e dois $(0,1 \%)$ com placebo relataram eventos adversos de ideação ou comportamento suicida (O’neil et al., 2017).

Desta forma, o estudo concluiu que diante de análise exploratória agrupada, não há motivo de preocupação em relação à segurança neuropsiquiátrica do tratamento com liraglutida $3,0 \mathrm{mg}$.

Em estudo post hoc de análise da segurança cardiovascular realizado para verificação de desfechos cardiovasculares com liraglutida 3,0 mg foi associado a um aumento médio do pulso e diminuição da pressão arterial sistólica. Embora o número geral de eventos cardiovasculares tenha sido baixo, o liraglutida não foi associado a um aumento da taxa de eventos cardiovasculares (Davies et al., 2018).

\subsection{Lorcaserina}

\subsubsection{Mecanismo de ação}

A lorcaserina é um agonista do receptor 2C da serotonina aprovado para o tratamento da obesidade em 2012. Seu mecanismo de ação proposto é a ativação seletiva dos receptores $2 \mathrm{C}$ da serotonina nos neurônios anorexigênicos no hipotálamo, inibindo a estimulação do apetite e promovendo a saciedade, embora seu mecanismo exato permaneça indefinido 
(nuffer; Trujillo \& Megyeri, 2016).

\subsubsection{Eficácia e segurança}

Um estudo de coorte retrospectivo, multicêntrico e observacional conduzido usando dados de um banco nacional de veteranos comparando os resultados clínicos de quatro medicamentos aprovados pela FDA (Food and Drug Administration) para perda de peso (orlistat, fentermina, fentermina-topiramato e lorcaserina), demonstrou que, para lorcaserina, fenterminatopiramato, orlistate, fentermina e MOVE! (programa de controle de peso desenvolvido na ocasião) isoladamente, a perda de peso percentual de 6 meses após pelo menos 20 semanas de intervenção foi de 3,6\%, 4,1\%, 2,1\%, 3,6\% e 1,6\%, respectivamente. Nesta análise, a utilização de lorcaserina mostrou-se inferior na perda de peso ao longo do tempo quando comparação com fentermina e fentermina-topiramato por exemplo, porém, mostrando-se superior frente a utilização de orlistat (Grabarczyk, 2018).

Em estudo que avalia a farmacocinética de lorcaserina de liberação prolongada para o tratamento da obesidade, é trazido três grandes ensaios clínicos de fase III (BLOOM, BLOSSOM e BLOOM-DM) que compararam o uso de lorcaserina 10mg duas vezes ao dia com placebo em adultos com idade entre 18-65 anos. Dois desses ensaios (BLOOM, BLOSSOM) incluíram indivíduos obesos ou com sobrepeso sem diabetes, enquanto o terceiro (BLOOM-DM) explorou a eficácia de lorcaserina em pacientes com sobrepeso ou obesos com diagnóstico de diabetes tipo 2 (Hurren; Dunham, 2017).

Sintetizando as informações obtidas dos três ensaios, a redução média da massa corporal da linha de base para esses estudos foi de 5,8\% com a dose aprovada de $10 \mathrm{mg}$, duas vezes ao dia, de lorcaserina, em comparação com uma redução média de peso de 2,2\% nos grupos de placebo. Em média, 44,1\% dos pacientes obtiveram 5\% de perda de peso e 20,5\% dos pacientes obtiveram $\geq 10 \%$ de perda de peso da linha de base no grupo de lorcaserina duas vezes ao dia, em comparação com 20,5\% e $7,3 \%$ dos pacientes com placebo atingindo $\geq 5 \% \mathrm{e} \geq 10 \%$ de perda de peso, respectivamente (Hurren \& Dunham, 2017).

Os efeitos adversos relatados no estudo mais comumente atribuídos a lorcaserina em ensaios de fase III incluem dor de cabeça, boca seca, constipação, tontura, fadiga e náusea. Em pacientes com diabetes, a hipoglicemia foi relatada em 29,3\% dos pacientes tratados com lorcaserina e 21,0\% dos pacientes tratados com placebo. Episódios raros de hipoglicemia grave foram associados ao uso concomitante de sulfonilureias (Hurren \& Dunham, 2017).

A lorcaserina é uma droga serotonérgica e, como tal, apresenta um risco de síndrome da serotonina quando combinada com outros medicamentos que atuam neste neurotransmissor, como inibidores seletivos da recaptação da serotonina (ISRSs), inibidores seletivos da recaptação da serotonina-norepinefrina ou drogas que pode afetar a depuração da serotonina, como lítio, tramadol ou antagonistas da dopamina (Nuffer, Trujillo\& Megyeri, 2016).

Visto isso, foram relatados efeitos adversos graves, incluindo síndrome da serotonina, síndrome neuroléptica maligna, comprometimento cognitivo, distúrbios psiquiátricos, priapismo, bradicardia e elevação da prolactina, de forma que lorcaserina deve ser usado com cautela em pacientes predispostos a essas doenças. Todos os 3 ensaios clínicos incluídos nesta revisão excluíram pacientes em tratamento para depressão ou ativamente tratados com ISRSs (Hurren \& Dunham, 2017).

\subsection{Novos fármacos para o tratamento da obesidade}

Além dos fármacos já estudados pelos grandes ensaios clínicos e já aprovados pelos órgãos reguladores para o tratamento da obesidade, seja ela pediátrica ou em adultos, existem atualmente muitos compostos sendo estudados como novas possibilidades terapêuticas para essa finalidade. No presente estudo trazemos algumas das possibilidades encontradas nesta revisão como opções terapêuticas que mostraram maior solidez nas conclusões dos estudos realizados, encontrando-se em processo de análise para comporem futuramente o rol de fármacos antiobesidade. 


\subsubsection{Exenatida}

Dentro dos agonistas do GLP-1 temos juntamente com a liraglutida, a exenatida. Ambas foram desenvolvidas pela primeira vez e, consequentemente, aprovadas para o tratamento de diabetes tipo 2 (DM2) em adultos, mostrando também efeitos sobre a redução de peso em adultos com obesidade (Weghuber et al., 2020).

Com relação ao mecanismo de ação da exenatida, este fármaco, assim como os demais agonistas do GLP-1, mostrou benefícios não só na perda de peso, mas no controle da glicose, pelo aumento da secreção de insulina pós-prandial dependente de glicose e inibição da secreção de glucagon, bem como melhora em outras comorbidades metabólicas, incluindo doença hepática gordurosa não alcoólica. Os mecanismos pleiotrópicos de ação que influenciam o apetite e a saciedade são pensados para estar relacionados às vias centrais, bem como periféricas (Weghuber et al., 2020).

De acordo com Weghuber et al, (2020) em ensaio clínico randomizado de seis meses, duplo-cego, paralelo e controlado por placebo, foi realizada avaliação em 44 pacientes de 10 a 18 anos, com IMC-SDS $>2,0$ ou IMC $>30 \mathrm{~kg} / \mathrm{m}^{2} \mathrm{de}$ acordo com a OMS. Os pacientes receberam intervenção no estilo de vida e foram randomizados para exenatida de liberação estendida $2 \mathrm{mg}$ ou placebo injeções subcutâneas administradas uma vez por semana. Foi observado que o IMC diminuiu significativamente no grupo exenatida em comparação com o grupo placebo durante o período de estudo. O IMC-SDS reduziu em 0,09 correspondendo a 3,0 kg. Além disso, a exenatida reduziu a circunferência da cintura em 3,2 cm.

Com relação à segurança, o número de eventos adversos (EAs) foi semelhante entre os dois grupos. Os EAs mais comuns foram gastrointestinais (flatulência, náusea, diarreia, constipação, dor abdominal, arrotos, vômitos e dor na boca), infecções (respiratórias, gastrointestinais e urinárias) e distúrbios do sistema nervoso (cefaleia, tonturas, síncope, tremor das mãos e parestesia). Considerando que os EAs gastrointestinais foram mais comuns no grupo exenatido (18 versus 10), os EAs de infecção (18 versus 20) e os EAs de distúrbio do sistema nervoso central (16 versus 13) foram semelhantes entre os grupos tratados com exenatida e tratados com placebo (Weghuber et al., 2020).

Desta forma, foi possível inferir que no presente estudo, o exenatida de liberação estendida melhora o controle glicêmico, o colesterol total e o colesterol LDL, ao mesmo tempo que reduz modestamente o IMC em adolescentes com obesidade. A administração de exenatida duas vezes ao dia e uma vez por semana demonstrou reduzir o IMC em adultos com diabetes mellitus do tipo 2. Foi observado ainda que a redução do IMC alcançada com exenatida é semelhante ao tratamento com orlistate ou cloridrato de metformina, já realizado em adolescentes com obesidade. Parece plausível que a dosagem menos frequente em comparação com exenatida duas vezes ao dia ou liraglutida uma vez ao dia possa ser mais atraente para os adolescentes.

\subsubsection{Melatonina}

A melatonina é amplamente produzida na glândula pineal, regulando o ritmo circadiano, e exercendo uma variedade de ações no organismo vivo. Entre essas funções, acredita-se que a melatonina tenha um efeito positivo no peso corporal e no metabolismo energético. Estudos em animais demonstraram que o tratamento diário com melatonina reduz o peso corporal, os níveis plasmáticos de leptina, adiponectina, triglicerídeos, colesterol, insulina e glicose (Amstrup et al., 2016).

Os mecanismos de ação para tais efeitos ainda são indefinidos, porém, sabe-se que a melatonina pode aumentar a expressão de proteínas morfogênicas ósseas (BMPs) 2 e 4, além de suprimir o receptor gama ativado por proliferador de peroxissoma (PRAR- c). Estas ações direcionam as células-tronco mesenquimais humanas (hMSC) para a linhagem celular osteogênica, resultando em diminuição da adipogênese da medula óssea. Além disso, a melatonina pode inibir o acúmulo de triglicerídeos induzido por ácido graxo, evitando assim um aumento nos triglicerídeos circulantes. As vias mediadas pelos receptores de melatonina também podem ser importantes, visto que estes demonstraram desempenhar um papel importante na sincronização dos principais órgãos envolvidos na regulação da glicose e agem negativamente na secreção de insulina 
(Amstrup et al., 2016).

Em um estudo duplo-cego controlado por placebo Amstrup, et al., (2016), foram randomizadas 81 mulheres na pósmenopausa para 1 ano de tratamento com melatonina (1 ou $3 \mathrm{mg}$ à noite) ou placebo. A composição corporal foi medida por DXA (densitometria por emissão de raios X de dupla energia). As medidas foram obtidas no início do estudo e após 1 ano de tratamento junto com leptina, adiponectina e insulina. Os marcadores da homeostase da glicose foram medidos no final do estudo. Em resposta ao tratamento, a massa gorda diminuiu no grupo da melatonina em $69 \%$ em comparação com o placebo. Um aumento significativo limítrofe na massa magra de 5,2\% foi encontrado no grupo de melatonina em comparação com o placebo $3,3 \%$ versus $1,9 \%$.

Após o ajuste para o IMC, a massa magra aumentou em 2,6\% no grupo da melatonina. Mudanças no peso corporal e no IMC não diferiram entre os grupos. A adiponectina aumentou significativamente na fronteira de $21 \%$ no grupo de melatonina em comparação com o placebo. Nenhuma mudança significativa foi observada para leptina, insulina ou marcadores da homeostase da glicose. Dessa forma foi observado um efeito possivelmente benéfico da melatonina na composição corporal e no metabolismo lipídico, já que 1 ano de tratamento reduz a massa gorda, aumenta a massa magra e está associado a uma tendência de aumento da adiponectina (Amstrup et al., 2016).

\section{Conclusão}

O tratamento de pacientes obesos e com sobrepeso baseia-se em mudanças comportamentais, dieta e exercícios, contudo, é sabido que a maioria das pessoas não consegue obter uma perda de peso adequada somente com modificações no estilo de vida. $\mathrm{O}$ tratamento antiobesidade então, é recomendado como um complemento às intervenções comportamentais em pacientes com IMC $\geq 30 \mathrm{~kg} / \mathrm{m}^{2}$ ou pacientes com IMC $\geq 27 \mathrm{~kg} / \mathrm{m}^{2}$ juntamente com uma ou mais comorbidades associadas.

A maioria dos medicamentos atualmente aprovados para o tratamento farmacológico da obesidade no Brasil, demonstrou em ensaios clínicos duplo-cegos, randomizados e controlados por placebo, a obtenção de uma perda de peso de pelo menos $\geq 5 \%$ em comparação com placebo, em pelo menos 35\% dos participantes do grupo de tratamento.

Em relação aos efeitos adversos, os eventos de natureza gastrointestinal pareceram ser consistentes e proeminentes em todos esses agentes, com exceção de sibutramina, que teve eventos de natureza cardiovascular mais proeminentes. Foram observadas taxas semelhantes de eventos adversos entre orlistate, liraglutida e lorcaserina, embora os pacientes em uso de liraglutida relatem os maiores efeitos colaterais gastrointestinais. Desta forma, fica evidente que os profissionais prescritores devem tomar suas decisões terapêuticas de forma individualizada e baseada em fatores específicos do paciente, incluindo medicamentos em uso concomitante, comorbidades associadas, grau de perda de peso desejada, bem como, tolerância aos eventos adversos.

No estudo realizado, as possibilidades de novos tratamentos farmacológicos para a obesidade que apresentaram maior solidez nos resultados de perda de peso, foram a exenatida e a melatonina, com eficácia demonstrada em ensaios clínicos. O perfil de efeitos adversos no caso da exenatida, mostrou-se semelhante ao perfil de liraglutida, porém com a vantagem de necessitar menor número de administrações, enquanto no caso da melatonina, ainda necessitam de mais estudos para uma melhor definição. Estudos futuros podem avaliar novas opções terapêuticas do controle da obesidade, em comparação com os representantes abordados neste estudo.

\section{Referências}

Agência Nacional de Saúde Suplementar (2017). Manual de diretrizes para o enfretamento da obesidade na saúde suplementar brasileira.

Amstrup, A. K., Sikjaer, T., Pedersen, S. B., Heickendorff, L., Mosekilde, L., \& Rejnmark, L. (2016). Reduced fat mass and increased lean mass in response to 1 year of melatonin treatment in postmenopausal women: A randomized placebo-controlled trial. Clinical Endocrinology, 84(3), $342-347$. 
Anvisa, Associação Nacional de Vigilância Sanitária. (2017). Dispõe sobre a comercialização e manipulação dos anorexígenos sibutramina, anfepramona, femproporex e mazindol.

Ard, J., Cannon, A., Lewis, C. E., Lofton, H., Vang Skjøth, T., Stevenin, B., \& Pi-Sunyer, X. (2016). Efficacy and safety of liraglutide 3.0 mg for weight management are similar across races: subgroup analysis across the SCALE and phase II randomized trials. Diabetes, Obesity nd Metabolism, 18(4), 430-435.

Arzola-Paniagua, M. A., García-Salgado López, E. R., Calvo-Vargas, C. G., \& Guevara-Cruz, M. (2016). Efficacy of an orlistat-resveratrol combination for weight loss in subjects with obesity: A randomized controlled trial. Obesity, 24(7), 1454-1463.

Costa, A. M. J, \& Duarte, S. F. P. (2017). Principais medicamentos utilizados no tratamento da obesidade e vias de ação: uma revisão sistemática. Id On Line Revista de Psicologia, 11(35), 199-209.

Davies, M. J., Aronne, L. J., Caterson, I. D., Thomsen, A. B., Jacobsen, P. B., Marso, S. P., \& Satiety and Clinical Adiposity-Liraglutide Evidence in individuals with and without diabetes (SCALE) study groups. (2018). Liraglutide and cardiovascular outcomes in adults with overweight or obesity: A post hoc analysis from SCALE randomized controlled trials. Diabetes, Obesity and Metabolism, 20(3), 734-739.

Gadde, K. M., Apolzan, J.W., Berthoud, H. R. (2018). pharmacotherapy for patients with obesity. Clinical chemistry, 64(1)118-129.

Grabarczyk, T. R. (2018). Observational comparative effectiveness of pharmaceutical treatments for obesity within the veterans health administration. Pharmacotherapy: The Journal of Human Pharmacology and Drug Therapy, 38(1), 19-28.

Hurren, K. M., \& Dunham, M. W. (2017). Pharmacokinetic drug evaluation of extended release lorcaserin for the treatment of obesity. Expert opinion on drug metabolism \& toxicology, 13(8), 891-896.

Instituto Brasileiro de Geografia e Estatística (IBGE). Pesquisa Nacional de Saúde 2019. Prevalência da obesidade entre adultos no Brasil. [Internet]. Rio de Janeiro: Instituto Brasileiro de Geografia e Estatística; 2020. https://biblioteca.ibge.gov.br/index.php/bibliotecacatalogo?view=detalhes\&id=2101764. Acesso em: 15 fev. 2021.

Isaacs, D., Prasad-Reddy, L., \& Srivastava, S. B. (2016). Role of glucagon-like peptide 1 receptor agonists in management of obesity. American journal of health-system pharmacy, 73(19), 1493-1507.

Lucchetta, R. C., Riveros, B. S., Pontarolo, R., Radominski, R. B., Otuki, M. F., Fernandez-Llimos, F., \& Correr, C. J. (2017). Systematic review and metaanalysis of the efficacy and safety of amfepramone and mazindol as a monotherapy for the treatment of obese or overweight patients. Clinics, 72(5), 317-324.

Nuffer, W., Trujillo, J. M., \& Megyeri, J. (2016). A comparison of new pharmacological agents for the treatment of obesity. Annals of Pharmacotherapy, 50(5), 376-388.

O'Neil, P. M., Aroda, V. R., Astrup, A., Kushner, R., Lau, D. C., Wadden, T. A., \& Satiety and Clinical Adiposity-Liraglutide Evidence in individuals with and without diabetes (SCALE) study groups. (2017). Neuropsychiatric safety with liraglutide $3.0 \mathrm{mg}$ for weight management: results from randomized controlled phase 2 and 3a trials. Diabetes, Obesity and Metabolism, 19(11), 1529-1536.

Pereira A. S. et al. (2018). Metodologia da pesquisa científica. UFSM.

Radaelli, M., Pedroso, R. C., \& Medeiros, L. F. (2016). Farmacoterapia da obesidade: Benefícios e Riscos. Saúde e Desenvolvimento Humano, 4(1), 101-115.

Shettar, V., Patel, S., \& Kidambi, S. (2017). Epidemiology of obesity and pharmacologic treatment options. Nutrition in Clinical Practice, 32 (4), $441-462$.

Silva, F. I. L., Rodrigues, G., Bonelli, O. A. (2019). O risco do uso dos anorexígenos disponíveis no brasil para o tratamento da obesidade. Revista saberes, $9(1)$.

Sociedade Brasileira de Endocrinologia e Metabologia (2010). Obesidade e sobrepeso: tratamento farmacológico. Brasília: Associação Médica Brasileira e Conselho Federal de Medicina.

Wadden, T. A., Tronieri, J. S., Sugimoto, D., Lund, M. T., Auerbach, P., Jensen, C., \& Rubino, D. (2020). Liraglutide 3.0 mg and intensive behavioral therapy (IBT) for obesity in primary care: the SCALE IBT randomized controlled trial. Obesity, 28(3), 529-536.

Weghuber, D., Forslund, A., Ahlström, H., Alderborn, A., Bergström, K., Brunner, S., \& Bergsten, P. (2020). A 6-month randomized, double-blind, placebo-controlled trial of weekly exenatide in adolescents with obesity. Pediatric obesity, 15(7), e12624. 